\title{
Design of output fluctuation smoothing strategy in photovoltaic power station
}

\author{
Yu Zhang ${ }^{l}$, Shanshan Shi ${ }^{1}$, Qixing Yang ${ }^{2}$, Chen Fang ${ }^{l}$, Qin Xu ${ }^{l}$, Yufei Wang 2,* \\ ${ }^{1}$ State Grid Shanghai M unicipal Electric Power Company, Shanghai, China \\ ${ }^{2}$ Shanghai University of Electric Power, Shanghai, China
}

\begin{abstract}
The output power of photovoltaic (PV) power station has strong fluctuation and randomness. Large-scale photovoltaic grid connection will affect the safe operation of power grid. In this paper, the smoothing strategy of PV output fluctuation is designed based on the adaptive moving average algorithm, which combined with the PV power prediction technology. The energy storage system compensates the difference between the grid-connected reference power and the actual generation power in real time, smoothing the grid-connected power of PV power station. Firstly, the relationship between the length of fixed sliding window and smoothness, as well as volatility in the moving average algorithm is explored to provide theoretical basis for subsequent parameter selection. Then, in order to enhance the adaptive performance of the algorithm, an adaptive moving average algorithm is proposed to dynamically adjust the length of the sliding window according to the actual power volatility. The PV power prediction curve is smoothed based on the algorithm so that the grid-connected reference power curve can be obtained. Finally, three typical weather conditions of sunny day, cloudy day and overcast day are taken as examples to simulate. The results show both feasibility and effectiveness of the strategy designed to smooth output fluctuation of PV power station.
\end{abstract}

\section{Introduction}

Affected by climate, geography and other environmental factors, PV power stations are unable to deliver electricity to the grid in a stable manner [1-3]. The energy storage system has flexible and fast two-way power regulation capability, which can smooth the PV power generation power according to the energy demand in real time, and reduce the adverse impact on the power grid. At present, using energy storage technology to design the corresponding control strategy has become an effective means to smooth the PV output fluctuation [45].

The control methods to suppress power fluctuations mainly include the low-pass filtering method [6], the moving average method [7] and the power prediction method [8]. Among them, as a simple and effective method for dynamic data processing, the moving average algorithm can eliminate the interference caused by periodic changes and random fluctuations and smooth the data sequence [9-11]. With the increase of the length of the sliding window, the filtering effect of the power curve becomes more obvious, but it may cause overcharge/discharge of the energy storage system, threatening the service life of the equipment. At the same time, the target value of filtering is limited by the input power to a large extent. When there is a sudden change of power fluctuation, the subsequent smoothing effect will be weakened due to the reduction of filtering performance. In this regard, domestic and foreign studies have continuously improved and optimized algorithms according to different control objectives and constraints. For example, The literature [12] considers the charged state and power volatility of energy storage batteries and adjusts the filtering bandwidth and weight coefficient of the moving average algorithm in real time to deal with the sudden change of wind power output. With the development of wind power industry in recent years, the smooth operation of wind power and the safety of power grid increasingly depend on the high precision of wind speed prediction technology, and the grid-connected power smoothing method combined with power prediction has been continuously studied in the field of wind power. The combination of the moving average algorithm and the power prediction technology can not only reduce the time delay caused by the algorithm, but also reduce the influence of the predicted power accuracy on the calculation results, thus achieving better smoothing effect [13].

PV power has similar or even stronger volatility than wind power, but it is still rare to apply the power prediction technology to the smooth fluctuation of $\mathrm{PV}$ output, and the research on the combined application of the moving average algorithm and the power prediction results is even less, which has a good research prospect. On this basis, the paper proposes to use the adaptive moving average algorithm to find the minimum sliding window length that meets the grid-connected standards,

\footnotetext{
Corresponding author: energystorage@ 126.com
} 
and then design the corresponding output fluctuation smoothing strategy based on the PV power generation power prediction curve, so that the energy storage system can absorb or release energy to realize PV power smoothing control according to the demand.

\section{PV power output smoothing based on adaptive moving average algorithm}

Set a non-stationary data set consists of $N$ actual measured data, and the mean value of each $m$ adjacent data interval is close to a constant, used to represent the endpoint or midpoint data of the $m$ adjacent data, which is regarded as the data value that has been smoothed. The formula of moving average algorithm is shown in (1), $m=2 n+1 ; k=n+1, n+2 \ldots, N-n$.

$$
y_{k}=\frac{1}{2 n+1} \sum_{k=-n}^{n} y_{k+1}
$$

The length of the sliding window is an important parameter of the moving average algorithm. The selection of the parameters will directly affect the smoothness of the curve and the power volatility of the data. A smaller length can improve the sensitivity of the energy storage system, but the stability of the PV power curve will be reduced, and fluctuations will impact the power grid obviously. A larger length can enhance the smoothness of PV power curve, so that the fluctuation range of PV power is small and basically stays within $10 \%$. However, a larger energy storage capacity is required correspondingly to increase the energy loss of the battery. The comparison curve of PV power smoothness and volatility when $L=3, L=6, L=9$ and fixed sliding window length is adopted by moving average algorithm is shown in Fig 1.

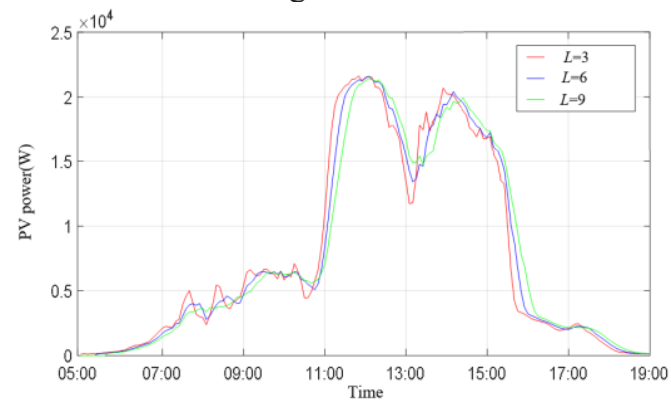

(a) Curve smoothness

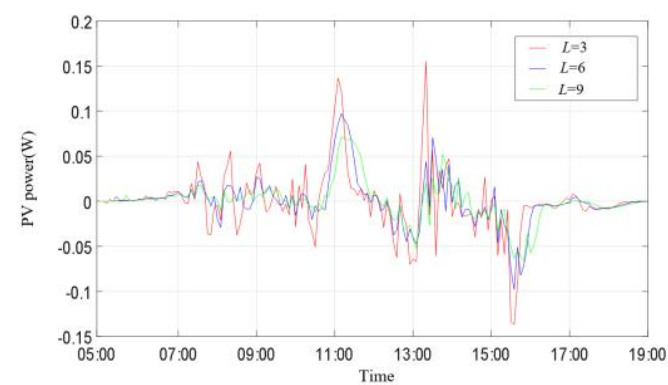

(b) PV power volatility

Fig. 1. PV power comparison curves under different sliding window lengths
It can be found that choosing the appropriate length of sliding window according to different actual power is the difficult point of applying the moving average algorithm. In this regard, an adaptive moving average algorithm is proposed to smooth the PV output, and the minimum sliding window length is found on the basis of meeting the grid-connected standards, so as to ensure the smooth effect of power curve and reduce the unnecessary energy loss of the energy storage system.

\subsection{Calculation process}

According to the Chinese National standard GB/T 19964-2012 "Technical Regulations on PV Power Station Access to Power System", the rate of change of active power of PV power station per minute shall not exceed $10 \%$ of installed capacity [14]. The formula of volatility $F(t)$ is shown in Equation (2), where $P(t)$ and $P(t-1)$ respectively represent the output power of PV power station at the time of $t$ and $(t-1) . P_{n}$ represents the installed capacity of PV power stations.

$$
F(t)=\frac{P(t)-P(t-1)}{\operatorname{Pn}} \times 100 \%
$$

The adaptive moving average algorithm dynamically adjusts the length of the sliding window by calculating the fluctuation degree of the actual PV power. If the volatility $F$ of the sampling point does not exceed $10 \%$, the next point will be calculated in a cycle. Otherwise, the moving average algorithm is used to smooth the actual power curve, and the length $L$ of the sliding window increases according to the number of cycles, and then the smoothen curve volatility is recalculated until all the sampling points meet the conditions and the output curve is finally output. Where, $N$ represents the total number of sampling points of PV power generation curve.

Set the sampling time resolution to 1 minute, and the specific calculation process is shown in Fig 2.

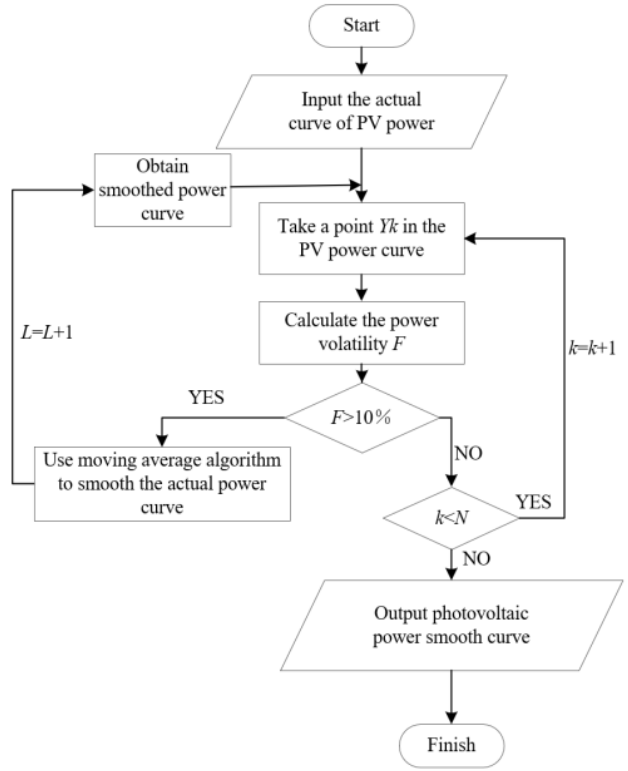

Fig. 2. The flow chart of calculation based on adaptive moving average algorithm 


\subsection{Simulation results and analysis}

Fig. 3 shows the smoothing effect of PV power station output using the adaptive moving average algorithm. At this point, $L=6$, that is, the adaptive selection of sliding window length is 6 minutes. As can be seen from the figure, on the basis that the power volatility just meets the requirements, the PV output smoothness effect is good, but there is a certain time delay between it and the actual PV generation curve. Compared with the fixed sliding window length, the improved algorithm can adjust the parameters flexibly according to the actual situation and has better adaptability.

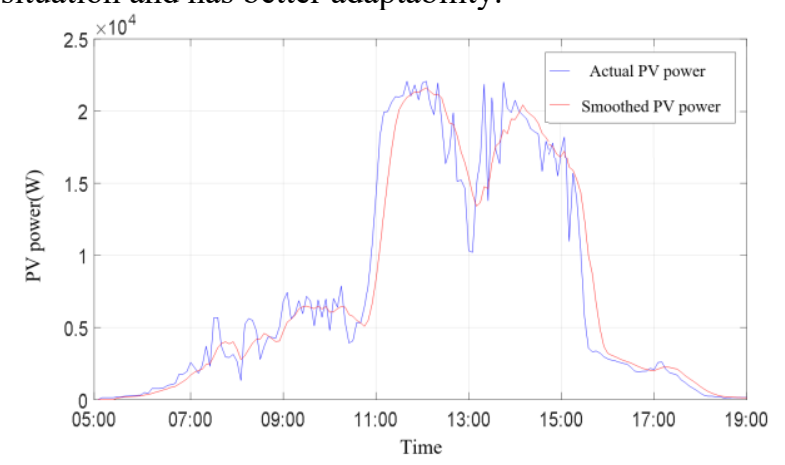

(a) Curve smoothness

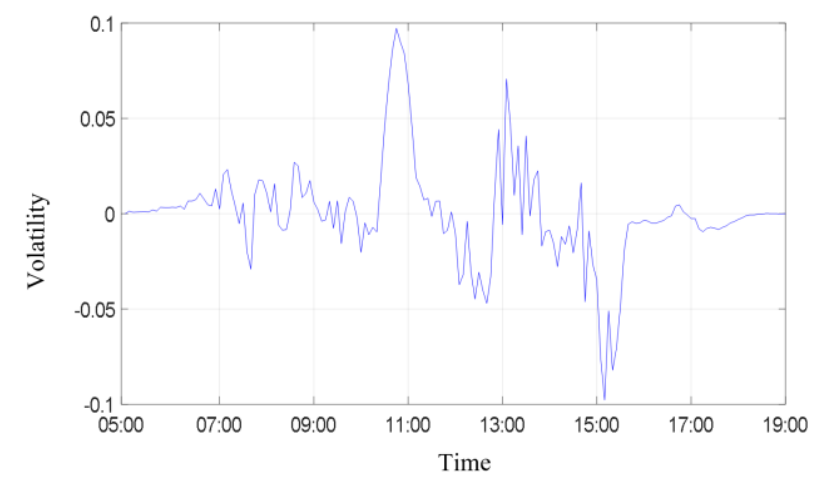

(b) PV power volatility

Fig. 3. The smooth effect diagram of PV power station output based on adaptive moving average algorithm

\section{The design of output fluctuation smoothing strategy based on PV power prediction}

When using the adaptive moving average algorithm to smooth the fluctuation, it does not take into account that the charging and discharging control of the energy storage battery at the present stage will be affected by the PV power in the future period, and the use of the moving average algorithm to smooth the time delay of output fluctuation will lead to the deviation of the action of the energy storage system. PV power prediction technology can obtain the prediction data in the future period in advance, and the combination of prediction curve and moving average algorithm can better eliminate the delay caused by moving average algorithm, and finally make the actual curve and smooth curve match well in time, so as to achieve the expected smoothing effect. For this reason, a PV power generation power smoothing strategy based on PV power prediction is proposed.

\subsection{Calculation process}

The output fluctuation smoothing strategy based on PV power prediction is to establish a predictive power model [15] through the theory of brain emotional learning neural network. Then, the PV power prediction curve is smoothed based on the adaptive moving average algorithm proposed above, and the result is used as the grid-connected power reference value input energy storage control simulation model. The energy storage system carries out charge and discharge control according to the power difference. When the actual generation power exceeds the grid-connected reference power, the energy storage system absorbs energy. When the actual generation power is lower than the reference power connected to the grid, the energy storage system releases energy, and finally achieves the PV output fluctuation suppression when the energy storage chargedischarge power allows.

Set the sampling time resolution to 1 minute and the predicted time resolution to 1 minute. The specific calculation process is shown in Fig 4.

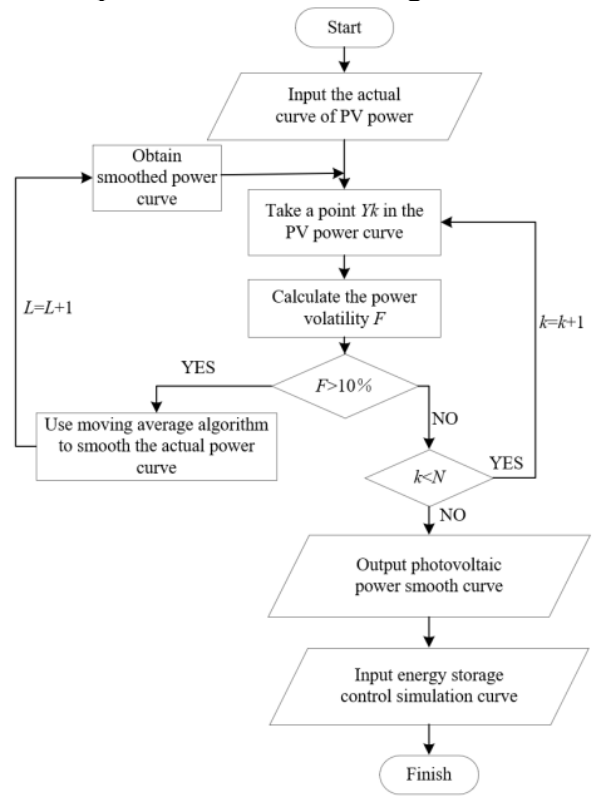

Fig. 4. The flow chart of the output fluctuation smoothing strategy

\subsection{The improvement of sliding window accuracy}

In order to make the final selected window length more accurate and reliable, measures to improve the accuracy of sliding window are proposed. Firstly, the predicted data with the predicted time resolution of 1 minute is imported into the module of "1-D Lookup Table" to generate curves. Then, the module of "To Workspace" is used to set the sampling time to $1 /(5 N-1)$. Data points with the time resolution of 12 seconds ( $1 / 5$ minutes) can be regained in the Workspace. When the length of the 
sliding window increases by 1 according to the number of cycles, the predicted data within the window increases by 12 seconds correspondingly to calculate the mean value and regain the smooth curve.

\subsection{Simulation results and analysis}

Three typical weather types (sunny day, cloudy day, overcast day) of a PV power station were selected for simulation. Considering that the PV power value at night and at the time of weak solar light intensity is 0 , data such as the predicted PV power of a certain day from 5 to 19 o'clock are selected and imported into Matlab/Simulink software platform. Set the sampling time resolution as 1 minute, the predicted time resolution as 12 seconds, and the initial sliding window length $L=1$ (12 seconds). The simulation results are shown as follows:

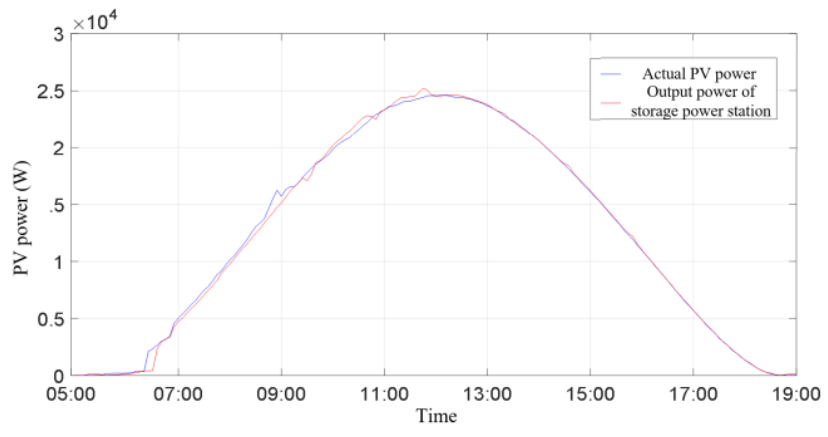

(a) Curve smoothness

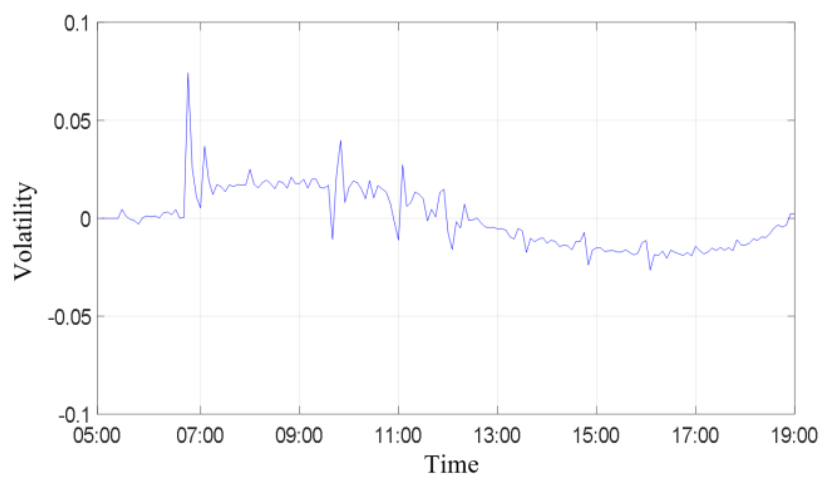

(b) PV power volatility

Fig. 5. Sunny day $(L=1)$

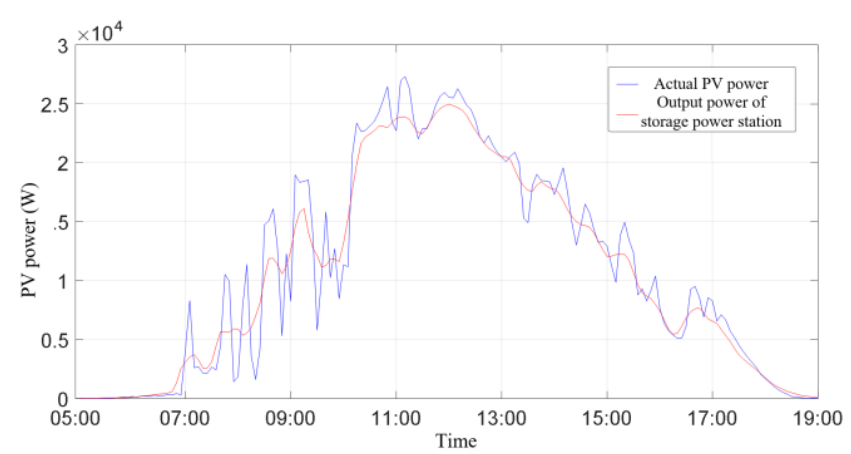

(a) Curve smoothness

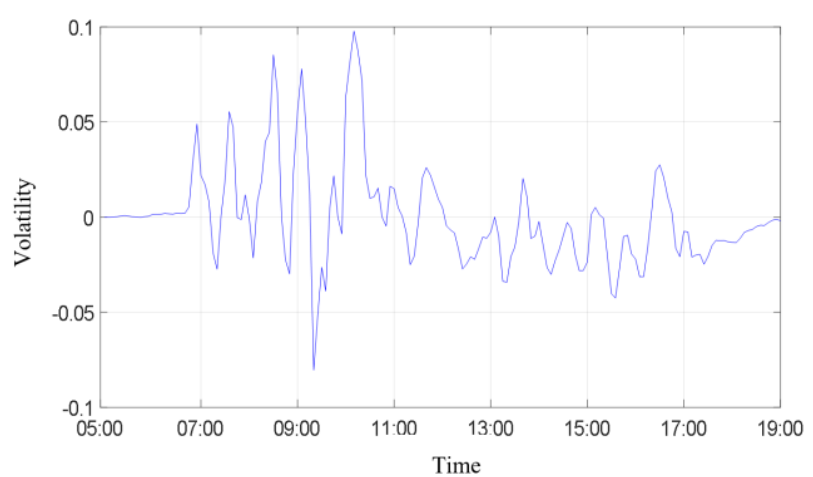

(b) PV power volatility

Fig. 6. Cloudy day $(L=24)$

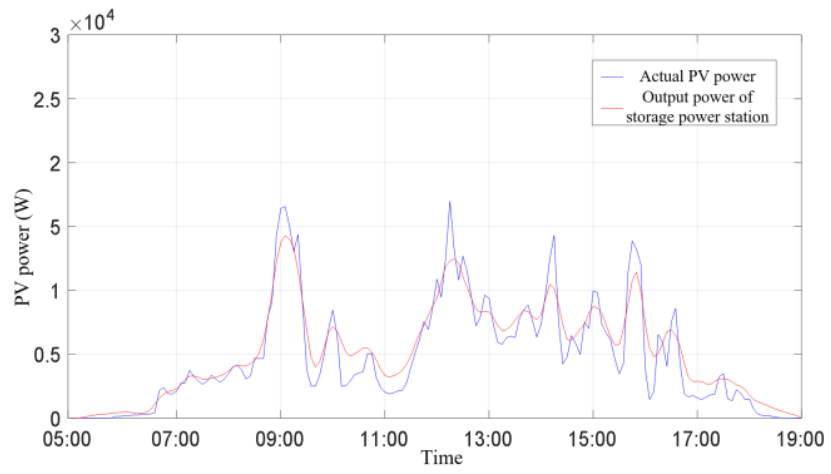

(a) Curve smoothness

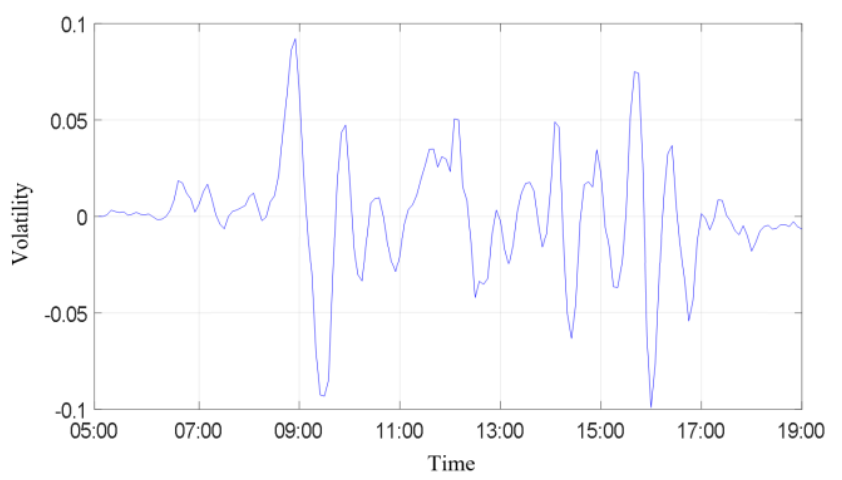

(b) PV power volatility

Fig. 7. Overcast day $(L=20)$

Among them, when the weather is sunny and there is sufficient light, the PV power generation curve is close to smooth. When the moving average algorithm is not used, the predicted power fluctuation rate is less than 0.08 , which can be directly input to the grid as gridconnected power; the PV power in cloudy weather fluctuates greatly with the change of cloud accumulation. The adaptive moving average algorithm is used to smooth the prediction curve until the grid-connected power volatility meets the requirements, when $L=24$, that is, the length of the sliding window is 4 minutes and 48 seconds; the solar radiation is weak in cloudy weather, and the PV power output value is also relatively small, the grid-connected power change is easier to meet the constraint conditions, at this time $L=20$, that is, the length of the sliding window is 4 minutes. 


\section{Conclusion}

To solve the problem of PV power station output fluctuation, the PV output fluctuation smoothing strategy is designed based on PV power prediction technology. The adaptive moving average algorithm selects the appropriate sliding window length according to the fluctuation degree of the predicted curve to smooth, and the energy storage system reduces the charge/discharge power loss as much as possible and ensures the timely and accurate operation of the system, so that the gridconnected power can be input into the power grid exactly meeting the fluctuation requirements. The simulation results verify the feasibility and effectiveness of the proposed strategy.

\section{References}

1. G.Y. Fei, Z.X. Lu, Y. Qiao, A utomation of Electric Power Systems, An Overview of Photovoltaic Energy System Output Forecasting Technology, 40, 140-151(2016)

2. S. Zhou, L. Zhou, M. Mao, X. Xi, 2020 IEEE International Conference on Big Data and Smart Computing (BigComp), Transfer Learning for Photovoltaic Power Forecasting with Long ShortTerm M emory Neural N etwork, 125-132(2020)

3. H. Zhou, Y. Zhang, L. Y ang, Q. Liu, et al. IEEE A ccess, Short-Term Photovoltaic Power Forecasting Based on Long Short Term Memory Neural Network and Attention Mechanism, 7806378074(2019)

4. Z.L. Ding, C.S. Du, C.H. Zhang, Journal of Power Supply, Configuration M ethod of Energy Storage to Suppress the PV Power Fluctuation, 24-30(2014)

5. P. Chung, J.C. W ang, C.Y. Chou, et al. 2020 8th International Electrical Engineering Congress (iEECON), An intelligent control strategy for energy storage systems in solar power generation based on long-short-term power prediction, 1$4(2020)$

6. P.C. Qiu, B.M. Ge, D.Q. Bi, Power System Protection and Control, Research on photovoltaic grid-connected power Generation power leveling control based on accumulator storage, 39, 2933(2011)

7. Q.Y. Chen, X.J. Li, X.J. Han, Electric Power Construction, A smooth wind power output control strategy for battery energy storage system based on moving average method and wind power volatility constraint, 34, 1-5(2013)

8. Y.F. Pan, J.H. Y ang, Computer Simulation, Power smoothing strategy for wave power generation system based on power prediction method, 35, 6872(2018)

9. F. Cheng, S. W illard, J. Hawkins, et al. 2012 IEEE Energytech, Applying battery energy storage to enhance the benefits of photovoltaic, 1-5(2012)

10. D. Xu, H. Cen, 2020 Asia Energy and Electrical Engineering Symposium (AEEES), A Hybrid Energy Storage Strategy Based Fuzzy Control to Suppress Power Fluctuation of Grid-Connected Photovoltaic Power System, 776-781(2020)

11. Y oga Sandi Perdana, S. M. M uyeen, A hmed AlDurra, et al. IEEE Transactions on Sustainable Energy, Direct Connection of SupercapacitorBattery Hybrid Storage System to the Grid-Tied Photovoltaic System, 1370-1379(2019)

12. M. Ding, J.F. Wu, C.Z. Zhu, Proc Chin Soc Elect Eng, Real-time smooth control strategy of energy storage system with soc adjustment function, 33, 2229(2013)

13. M.Y. Lei, Z.L. Y ang, Y.B. W ang, Transactions of China Electrotechnical Society, Study on energy storage control technology in optical/storage hybrid system, 31, 86-92(2016)

14. W.T. Jin, H.M. Ma, J.L. Li, Mod Electr Power, Research on the control method of photovoltaic power fluctuation of battery energy storage system, 30, 21-26(2013)

15. E. Lotfi, A. K hosravi, M. A kbarzadeh-T, et al. 2014 IEEE International Conference on Systems, Wind power forecasting using emotional neural networks, 311-316(2014) 